\title{
Rock Moisture Recognition by Combining AE with Musical-staff-inspired Model
}

\author{
Jingyu Jiang ${ }^{a^{*}}$, Wei Zheng and Kai Tao \\ Key Laboratory for Optoelectronic Technology and System of the Education Ministry of China, \\ College of Optoelectronic Engineering, 400044 Chongqing University Chongqing, China \\ ajiangjingyu286012@163.com
}

Keywords: Rock moisture; acoustic emission; musical-staff-inspired signal processing method

\begin{abstract}
Mechanical properties of rocks change under hydrous condition, which easily causes instability and failure of rock, and triggers a disaster. Questions about determining the effect of moisture on the acoustic emission characteristics of rocks, and establishing an automatic identification model loom large at present. In this study, a large number of acoustic emission data were collected during the rupture of rocks with different moisture contents, and the data were turned into data that are composed of musical parameters by a musical-staff-inspired model which reflect AE characteristics of water-filled rocks. Through training these data with a curves similarity determination method, the measured rock could be classified into the corresponding moisture group. Results of experiments indicate success of recognition of rock moisture.
\end{abstract}

\section{Introduction}

Some physical properties of rocks change under hydrous condition could easily causes instability and failure of rock, even triggers a disaster. Rock damage caused by hydration weakening, which is the primary cause of engineering rock failure, is more serious than those generated by external mechanical factors[1]. Thus, research on determining the rock moisture content has important implications for geotechnical engineering.

As an effective non-destructive method that has dynamic monitoring coverage, high sensitivity and other superiorities, acoustic emission (AE) is widely used in geotechnical engineering field[2-3]. But there lack a distinguishing method for rock moisture content that can tightly combine moisture content and AE characteristics. Considering the difficulty of establishing an automatic analysis model in terms of large amount of data and many characteristic parameters of AE signals, the present study utilizes the musical-staff signal processing model to dispose AE signal processing method. The developed model and AE technology were then used to evaluate rock moisture content.

\section{Musical-staff-inspired model}

The common geotechnical characteristics of $\mathrm{AE}$ signal, including amplitude, event duration, rising time, AE event count, threshold value, and energy envelope[4], are shown in Figure 1.

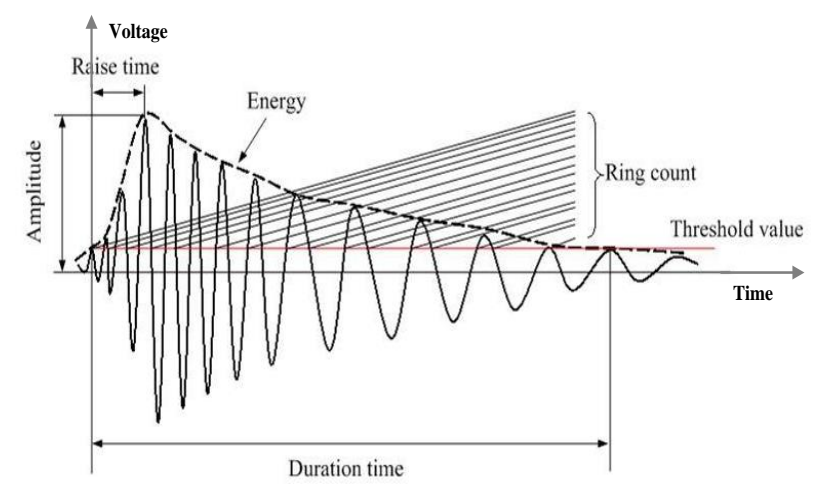

Figure. 1. Definition of the AE characteristic parameters in a simplified waveform. 
In a common method to analyze the characteristics of $\mathrm{AE}$ signal, rising time reflects the $\mathrm{AE}$ signal changing rate, and energy consumption reflects the energy level of AE signal. These parameters describe a certain feature of AE signal within a short time and are incapable of reflecting the global integrated characteristics, such as signal contour and changing trends within a wide time domain.However, the aforementioned parameters lack the standard expressions of discrete signals. Thus, a musical-staff-inspired signal processing method is applied in this study.

A musical staff is a set of five horizontal lines and four spaces that represent different musical pitches, as shown in Figure. 2.

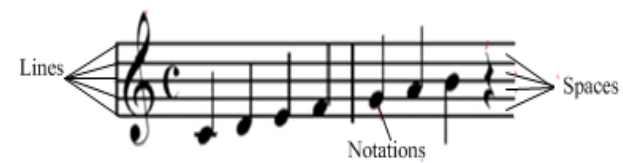

Figure. 2. Musical Staff.

In this method, the envelope line is quantified by planning musical staff. The pitch replaces amplitude of original signal while the phonetic denotes the transition rate. The entire process is depicted in Figure. 3.
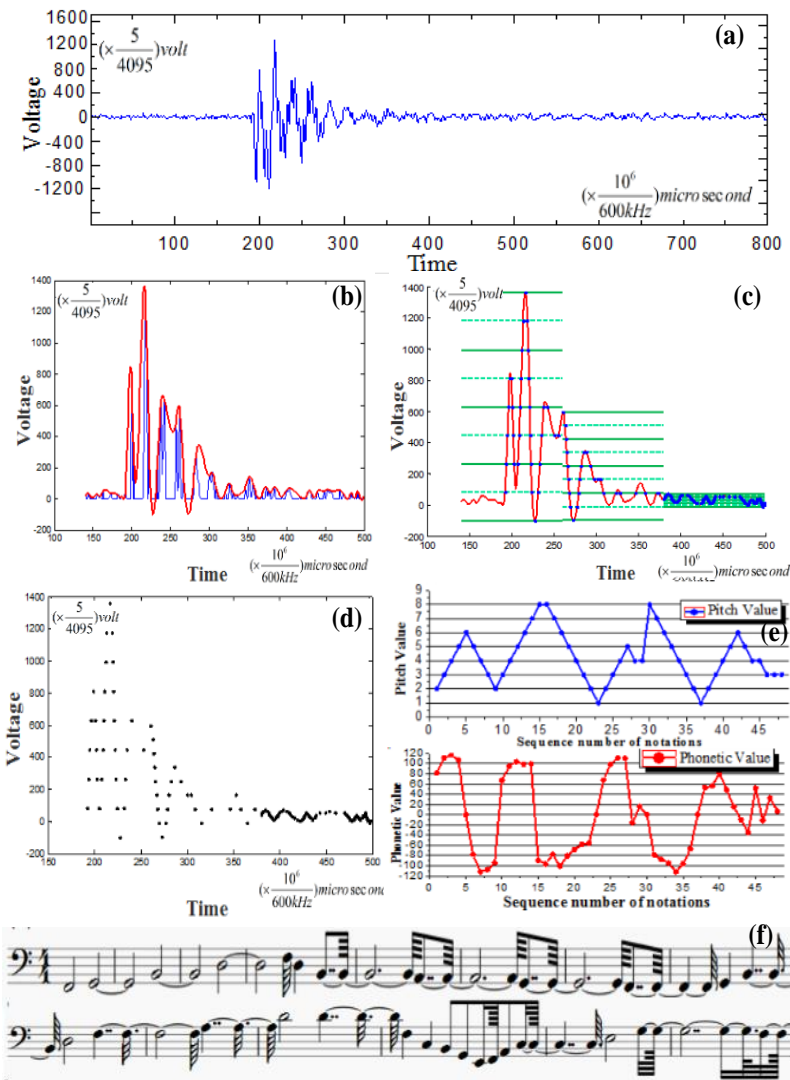

Figure. 3. Procedure of the musical-staff-inspired model.

Experiment showed that the musical-staff-inspired model can reach a fidelity value as high as $98.54 \%$ in reproducing the information of the original signal while achieving $16.86 \%$ of data compression ratio; these characteristics are convenient for storage and management[5].

\section{Similarity recognition method}

Since the pitch and phonetic curves are figured out, Fréchet arithmetic, the similarity recognition method is used to realize the discrimination to the moisture of rock.

The Fréchet distance was originally defined by Maurice Fréchet in 1906 as a measure of similarity between two parametric curves; it is widely used to measure similarity between curves. The Fréchet distance is intuitive and takes into account global features of the curves instead of local ones, such as their vertices. Given two curves $A=<a_{1}, \ldots, a_{m}>$ and $B=<b_{1}, \ldots, b_{n}>, d_{F}^{1}(A, B)$ exhibits 
the discrete Fréchet distance between the vertices, and $d_{F}^{2}(A, B)$ represents the discrete Fréchet distance between the nadirs. Then, let $\varepsilon$ be a threshold. Using $\left|d_{F}^{1}(A, B)-d_{F}^{2}(A, B)\right| \leq \varepsilon, A$ and $B$ are affirmed to be similar[6].

3.1 Normalizing the phonetic curve. Interpolation is included in the procedure of the structure of envelope to eliminate the effects on similarity recognition. Then, the musical-staff-inspired model would compress or stretch the envelope to avoid the appearance of gigantic difference in pitch value. Nonetheless, the variation trend of phonetic value can be approximate, whereas the magnitudes vary from each other because of existing difference of the magnitude of envelope of an AE signal. Thus, the dissimilarity of phonetic curve may emerge even through the rocks hold identical moisture content. To avoid the impact of this phenomenon on the setting procedure of $\varepsilon$, the numerical range of phonetic value must be unified before comparing the similarity of curves. This objective is achieved using a maximum difference normalization method, as follows:

$$
q_{i}^{\prime}=\frac{q_{i}-q_{\min }}{q_{\max }-q_{\min }}
$$

where $q_{i}^{\prime}$ is the normalized magnitude of the curve, and $q_{i}$ is the value of phonetic curve at its sampling site. $q_{\min }$ is the minimum of the curved section and $q_{\max }$ is the maximum. As such, the oversized gap of magnitude, which causes erroneous judgment of Fréchet similarity recognition, can be solved to some extent.

\subsection{Model training}

a) A large number of $\mathrm{AE}$ data from the rock, which are grouped on their moisture are collected;

b) These AE data are resolved into pitch and phonetic curves through the music-staff-inspired model;

c) Then the pitch and phonetic curves are contrasted among the groups by the discrete Fréchet similarity recognition, and the variance ( $\sigma_{P i}$ for pitch, $\sigma_{P h}$ for phonetic) is obtained based on the results of each comparison among groups. $\left[\bar{\varepsilon}_{p i}-\sigma_{p i}, \bar{\varepsilon}_{p i}+\sigma_{p i}\right]$ (or $\left[\bar{\varepsilon}_{p h}-\sigma_{p h}, \bar{\varepsilon}_{p h}+\sigma_{p h}\right]$ ) is considered the threshold and treated as the training group.

\subsection{Model procedure}

a) The AE data from the rock whose size are identical with that in the training group with unknown moisture are collected, and they are treated as test group;

b) Resolving the AE data into pitch and phonetic curves through the music-staff-inspired model;

c) The comparisons between the musical data of test group and training group are made by using Fréchet arithmetic. If the comparative result (a value of the Fréchet distance) of the contrast between the sample and either pair of data lies in the threshold $\left(\left[\bar{\varepsilon}_{p i}-\sigma_{p i}, \bar{\varepsilon}_{p i}+\sigma_{p i}\right]\right.$ and $\left.\left[\bar{\varepsilon}_{p h}-\sigma_{p h}, \bar{\varepsilon}_{p h}+\sigma_{p h}\right]\right)$ and the number of notations also lies in the threshold $\left(N_{x}=\left[\bar{n}_{x}-\sigma_{x}, \bar{n}_{x}+\sigma_{x}\right]\right)$, then the moisture of the sandstone sample is approximate to the moisture of the sandstone in the compared training groups.

\section{Experiment and result analysis}

4.1 Model experiment. Three groups (50 samples in each group) of yellow sandstones with same dimensions are disposed by the procedure in 3.1. the threshold values, which serve as the comparative result of training group, are shown in Table 1. 
Table 1. Threshold values of training group.

\begin{tabular}{|c|c|c|}
\hline & Pitch's threshold Value & Phonetic's threshold Value \\
\hline $0 \%$ Moisture & {$[2.998,3.2182]$} & {$[0.34126,0.37072]$} \\
\hline $25 \%$ Moisture & {$[3.368,3.8012]$} & {$[0.34883,0.38112]$} \\
\hline $50 \%$ Moisture & {$[4.0386,4.3928]$} & {$[0.37256,0.41462]$} \\
\hline $75 \%$ Moisture & {$[3.6712,3.8125]$} & {$[0.36259,0.37866]$} \\
\hline $100 \%$ Moisture & {$[3.5903,3.7402]$} & {$[0.3508,0.36912]$} \\
\hline
\end{tabular}

A yellow sandstone sample, whose size is same as the samples in the training group with known moisture of $95 \%$, is analyzed by the moisture determination model. The result in Fig. 11 shows that the pitch and phonetic curves are approximate to those of the sample sandstone in the training group with $100 \%$ moisture.

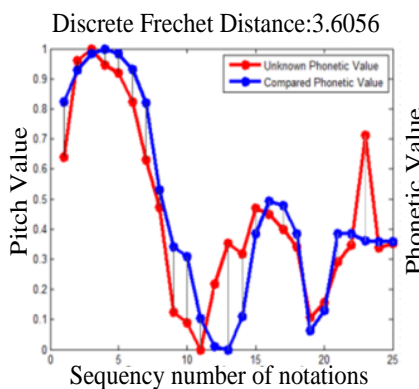

(a)

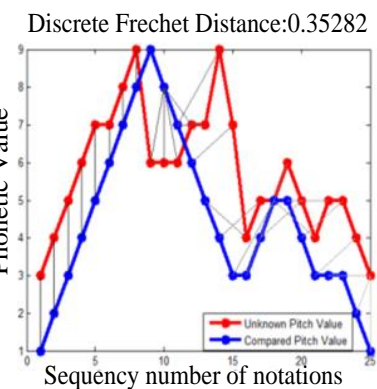

(b)

Figure. 4 Fréchet distance of the pitch and phonetic values of the measured sandstone.

This picture depicts that the Fréchet distance between the measured sandstone and the sandstone sample and the notations' number are lie in the threshold of the training group whose moisture are $100 \%$. Therefore, the moisture of measured sandstone can be considered to approximate to $100 \%$.

\subsection{Experiment results}

The identical procedure that same as 4.1 occur in three groups(20 samples in each groups) of yellow sandstones with the diverse moisture of $0 \%, 50 \%, 75 \%$ and $100 \%$. According to the results, false acceptance number (FAN, i.e., the number of samples whose moisture content is identical to that of the training group but do not belong to one category), false rejection number (FRN, i.e., the number of samples whose moisture content is different from that of the training group but belong to the same category), and correct classification number $(\mathrm{CCN})$ are utilized as the assessment indexes for model verification. The inspection result is shown in Figure. 5. 


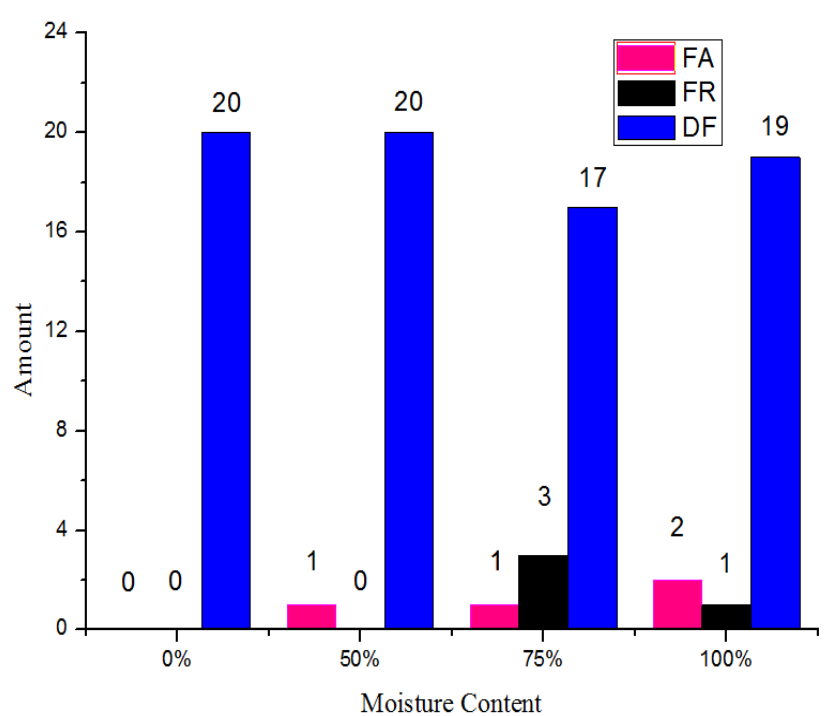

Figure. 5. FAN, FRN and CCN of classification result about each differently moisture.

Figure. 5 shows a preferable classification result of the sandstones with $0 \%$ moisture, which successfully classifies the 20 measured sandstones with $0 \%$ moisture. The 20 measured sandstones with $50 \%$ moisture are correctly classified, but a measured sandstone sample with verifiable moisture of $75 \%$ is falsely accepted. Moreover, 17 measured sandstones with $75 \%$ moisture are correctly included in one category, whereas 3 samples are rejected and 1 with $100 \%$ moisture is adopted. As for the group with $100 \%$ moisture, 19 samples are accepted, 1 sample is erroneously excluded, and 2 samples with $75 \%$ moisture are accepted.

The preceding analysis indicates that the moisture determination model is capable of determining the quantitative relationship between moisture and $\mathrm{AE}$ of the sandstone samples and can realize the automatic determination of moisture contents of stones.

\section{Conclusions}

In allusion to the puzzle that the existing engineering technology lack of a automatic identification technique to discriminate the moisture of rock, this study bases the phenomenon that the characteristics of $\mathrm{AE}$ signals from stone with diverse moisture are different, adopt a neotype musical-staff-inspired model to transform AE signal to a curve consists of discrete pitch and phonetic value. Fréchet similarity comparison algorithm is combined with contrast about the number of notations to inspect the similarity degree of pitch curve, phonetic curve. As a result, the moisture determination model is capable to reveal the quantitative relationship between moisture and $\mathrm{AE}$ of stone, and this research achievement could provide technical assistance to predict and prevent the geological disaster, such as permeable rock, collapse of mine, etc.

\section{Acknowledgments}

The authors would like to thank the project supported by the National Natural Science Foundation of China (Grant No. 61573073), the Chongqing City frontier general and applied basic research projects (Project No. cstc2015jcyjA40008) and the Fundamental Research Funds for the Central Universities (Project No. 106112015CDJXY120014).

\section{References}

[1] E.Z. Lajtai, R.H. Schmidtke, L.P. Bielus. Int J Rock Mech Min. The effect of water on the time-dependent deformation and fracture of a granite. 24[4], 247-255(1987)

[2] M.E. El-Alej, M Corsar, D Mba. Monitoring the presence of water and water-sand droplets in a horizontal pipe with Acoustic Emission technology. Appl Acoust 82[1], 38-44(2014) 
[3] Z.A. Moradian, G Ballivy, P Rivard. Rousseau B. Evaluating damage during shear tests of rock joints using acoustic emissions. Int J Rock Mech Min 47[4], 590-598(2010)

[4] A. Carpinteri, G Lacidogna, S Invemizzi. Stability of the vertical bearing structures of the Syracuse Cathedral: experimental and numerical evaluation. Mater Struct 42[7], 877-888(2009)

[5] W Zheng, C Wu. An information processing method for acoustic emission signal inspired from musical staff. Mech Syst Signal Pr 66[1], 388-398(2016)

[6] A.F. Cook, A Driemel, J Sherette, C Wenl. Computing the Fréchet distance between folded polygons. Comp Geom 50[1], 1-16(2015) 